\title{
NUMERICAL RECONSTRUCTION OF MULTIPHASE FLOWS WITH PRESCRIBED TOTAL MOMENTUM*
}

\author{
MaRJolaine PUel ${ }^{1}$
}

\begin{abstract}
We want to reconstruct numerically a two-phase flow knowing some partial information such as total density or total momentum using the least action principle. For that, we use the augmanted Lagrangian method.
\end{abstract}

AMS Subject Classification. 65M60.

\section{INTRODUCTION}

Let us consider a multiphase flow moving in a periodic domain $D$ during a finite interval of time $[0, T]$. We want to reconstruct the density $c(t, x, a) \geq 0$ and the velocity $v(t, x, a)$ for each phase $a$, at each point and each time knowing some partial information such as total density or total momentum of all phases. Among all admissible solutions, we select the flow that minimizes the total kinetic energy defined by

$$
K(c, m)=\frac{1}{2} \sum_{a=1}^{N} \int \frac{|m|^{2}}{c} d t d x=\frac{1}{2} \sum_{a=1}^{N} \int|v|^{2} c(t, x, a) d t d x, \text { with } m=c v .
$$

Related problems occur in Lagrangian data assimilation in oceanography [S]. The case when the total density of all phases is known and uniform in space and time, has been treated by Y. Brenier in [BR]. Then the optimal multiphasic flow satisfies some generalized incompressible Euler equations. In our study, we consider the case when the total momentum of all phases is zero, the optimal density and velocity must minimize $K(c, m)$ subject

\footnotetext{
Keywords and phrases: two-phase flow, least action problem, augmented Lagrangian

* I thank the CEMRACS and specially Stéphane CORDIER and Frédéric COQUEL for having given me the oportunity to participate to this summer-school.

${ }^{1}$ Laboratoire d'Analyse Numérique, Université Paris 6, 75 252, France

e-mail: mpuel@ann.jussieu.fr
}

(c) EDP Sciences, SMAI 2001 
to

$$
\begin{aligned}
\text { conservation of mass } & \partial_{t} c+\nabla \cdot m=0 \text { for all } a \\
\text { incompressibility } & \sum_{a=1}^{N} c(t, x, a)=1 \\
\text { nul total momentum } & \sum_{a=1}^{N} m(t, x, a)=0 .
\end{aligned}
$$

We have shown in $[\mathrm{P}]$, for a continuum of phase, the optimal multiphase flow satisfies a system of equations involving a Lorentz force and equivalent to the Vlasov-Maxwell system in an appropriate asymptotic regime.

Here, we treat numerically the same problem for a two-phase flow in a two-dimensional periodic box. Since the problem with two phases is simpler, we will not use the general result of $[\mathrm{P}]$. We explain here how we treat directly this particular case.

\section{Formulation OF THE PROBLEM}

For a diphasic flow, some constraints can be simplified and we do not have to use a Lagrange multiplier for each constraint. We then have the following proposition

Proposition 2.1. The problem described above can be solved through the following minimization:

$$
\sup _{c, m} \inf _{\phi, a, b} L(c, m, \phi, a, b)
$$

with

$$
L(c, m, \phi, a, b)=\int\left[\frac{\left(a+\frac{|b|^{2}}{2}\right)^{2}}{2|b|^{2}}+c\left(\partial_{t} \phi-a\right)+m \cdot\left(\nabla_{x} \phi-b\right)\right] d t d x+\int\left[\phi(0) c_{0}-\phi(T) c_{T}\right]
$$

Proof: Indeed, let us denote by $(c, m)=\left(c_{1}, m_{1}\right)$ the concentration and momentum fields of phase 1 and $\left(c_{2}, m_{2}\right)$ those of phase 2 . Constraints $(2)$ and (3) can be written

$$
m_{1}+m_{2}=0 \text { and } c_{1}+c_{2}=1
$$

The kinetic energy is then

$$
K(c, m)=\int \frac{1}{2} \frac{|m|^{2}}{c(1-c)} d t d x
$$

and the minimization problem involve the following Lagrangian

$$
\tilde{L}(c, m, \phi)=K(c, m)-\int\left(\partial_{t} \phi c+\nabla_{x} \phi \cdot m\right) d t d x-\int\left[\phi(0) c_{0}-\phi(T) c_{T}\right]
$$

Indeed, the introduction of the Lagrange multiplier $\phi$ forces $(c, m)$ to satisfy $(1)$ and the initial and final conditions.

To obtain the Lagrangian (4), we use the following proposition 
Proposition 2.2. the kinetic energy can be written by

$$
K(c, m)=\sup _{(a, b) \in C\left([0, T] \times D, \mathbb{R}^{1+d}\right)} \int\left[c a+m \cdot b-\frac{\left(a+\frac{|b|^{2}}{2}\right)^{2}}{2|b|^{2}}\right] d t d x
$$

Proof: Let us do the calculation :

$$
\begin{aligned}
I & =\sup _{(a, b)} \int\left[c a+m \cdot b-\frac{\left(a+\frac{|b|^{2}}{2}\right)^{2}}{2|b|^{2}}\right] d t d x \\
& =\sup _{b}\left(\int m \cdot b d t d x+\sup _{a}\left(\int\left(-\frac{a^{2}}{2|b|^{2}}+a\left(c-\frac{1}{2}\right)-\frac{|b|^{2}}{8}\right) d t d x\right)\right) .
\end{aligned}
$$

We obtain after the minimization with respect to $a$ wich can be done pointwise

$$
\begin{aligned}
I & =\sup _{b} \int\left[m \cdot b-\frac{|b|^{2}}{8}+\left(c-\frac{1}{2}\right)^{2} \frac{|b|^{2}}{2}\right] d t d x \\
& =\sup _{b} \int\left[c(c-1) \frac{|b|^{2}}{2}+m \cdot b\right] d t d x \\
& =-\int \frac{|m|^{2}}{4 \frac{c(c-1)}{2}} d t d x=\int \frac{|m|^{2}}{2 c(1-c)} d t d x=K(c, m) .
\end{aligned}
$$

We replace in (5) $K(c, m)$ by (6), and we obtain a new expression for the Lagrangian wich becomes (4) after a change of sign.

Let us remark that $(c, m)$ can be seen as a Lagrange multiplier for the constraint

$$
\partial_{t} \phi=a \text { and } \nabla_{x} \phi=b
$$

This remark allows us to use the Augmented Lagrangian method introduced in [FG] to solve the problem.

\section{The augmented Lagrangian Algorithm}

\subsection{Description of the algorithm}

In this part, we present the numerical algorithm used to solve the problem. We follow the one used by J.D. Benamou and Y. Brenier in [BB] to solve the following mass transport problem that we call (TM1):

Find a map $M: \mathbb{R}^{d} \rightarrow \mathbb{R}^{d}$ which transports a density $\rho_{0}$ to a density $\rho_{T}$, in the sense that for all borel set $A$

$$
\int_{M^{-1}(A)} \rho_{0}(x) d x=\int_{A} \rho_{T}(x) d x
$$

minimizing the so-called transportation cost

$$
\int_{\mathbb{R}^{d}}|x-M(x)|^{2} \rho_{0}(x) d x
$$


The authors of $[\mathrm{BB}]$ show that problem (TM1) can be solved introducing a time variable and solving the problem (TM2) looking for

with $\rho \geq 0$ and $v$ satisfying

$$
\inf _{\rho, v} T \int_{\mathbb{R}^{d}} \int_{0}^{T} \rho(t, x)|v(t, x)|^{2} d t d x
$$

$$
\begin{gathered}
\partial_{t} \rho+\nabla \cdot(v \rho)=0 \\
\rho(0, x)=\rho_{0}(x) \text { and } \rho(T, x)=\rho_{T}(x) .
\end{gathered}
$$

The problem (TM2) is very closed to our problem and therefore can be solved in the same way. But here, the constraints lead to another shape for the kinetic energy.

Let us now describe the algorithm.

We introduce the Augmented Lagrangian

$$
L_{r}(c, m, \phi, a, b)=L(c, m, \phi, a, b)+\frac{r}{2}\left(\int\left(\partial_{t} \phi-a\right)^{2} d t d x+\int\left|\nabla_{x} \phi-b\right|^{2} d t d x\right)
$$

and we solve numerically the problem

$$
\sup _{(c, m)} \inf _{(\phi, a, b)} L_{r}(c, m, \phi, a, b) .
$$

It is shown in the book of Fortin and Glowinski [FG] that problems with $r \geq 0$ or with $r=0$ have the same solution.

The numerical algorithm can be decomposed in 3 steps.

First step: we look for $\phi^{n}$ so that

$$
L_{r}\left(c^{n}, m^{n}, \phi^{n}, a^{n-1}, b^{n-1}\right) \leq L_{r}\left(c^{n}, m^{n}, \phi, a^{n-1}, b^{n-1}\right) \forall \phi .
$$

This step corresponds to solve a Poisson equation in $\phi^{n}$ with periodic boudary conditions in space and Neumann boudary conditions in time

$$
\begin{aligned}
& \Delta_{t, x} \phi^{n}=\partial_{t}\left(c^{n}-r a^{n-1}\right)+\nabla_{x} \cdot\left(m^{n}-r b^{n-1}\right) \text { in }[0, T] \times D \\
& -\partial_{t} \phi^{n}(0, x)=c^{n}(0, x)-r a^{n-1}(0, x)-c_{0} \text { in } D \\
& \partial_{t} \phi^{n}(T, x)=c^{n}(T, x)-r a^{n-1}(T, x)-c_{T} \text { in } D .
\end{aligned}
$$

This equation is solved by Fourier transforming the usual 5 points centered difference scheme in space and then inverting the resulting tridiagonal system in time for each Fourier mode.

Second step: we want to find $a^{n}$ and $b^{n}$ so that

$$
L_{r}\left(c^{n}, m^{n}, \phi^{n}, a^{n}, b^{n}\right) \leq L_{r}\left(c^{n}, m^{n}, \phi^{n}, a, b\right) \forall a, b .
$$

We will see in part 3.2 that this minimization is in fact a minimization on a monodimensional variable $|b|$.

Third step: we update the Lagrange multiplier

$$
\begin{gathered}
c^{n+1}=c^{n}+r\left(\partial_{t} \phi^{n}-a^{n}\right) \\
m^{n+1}=m^{n}+r\left(\nabla_{x} \phi^{n}-b^{n}\right) .
\end{gathered}
$$

To develop numerically this method, we modify the program written in [BB]. The first and the third steps are not modified and we explain the second step in the next part. 


\subsection{Study of the second step}

We will prove that for the second step, we just have to find a root of a polynomial.

We are looking for $a$ and $b$ wich are solutions of the minimization problem

$$
\begin{aligned}
& \inf _{b}\left(\int\left(\frac{|b|^{2}}{8}+\frac{r}{2}\left(\nabla_{x} \phi^{n}-b\right) \cdot\left(\nabla_{x} \phi^{n}-b\right)+m^{n} \cdot\left(\nabla_{x} \phi^{n}-b\right)+c^{n} \partial_{t} \phi^{n}+\frac{r}{2}\left(\partial_{t} \phi^{n}\right)^{2}\right) d t d x\right. \\
& +\inf _{a}\left(\int\left(a^{2}\left(\frac{1}{2|b|^{2}}+\frac{r}{2}\right)+a\left(\frac{1}{2}-r \partial_{t} \phi^{n}-c^{n}\right)\right) d t d x\right) .
\end{aligned}
$$

After an explicit pointwise calculation, the optimum $a^{n}$ satisfies

$$
a^{n}=-\frac{\left(\frac{1}{2}-r \partial_{t} \phi^{n}-c^{n}\right)}{2\left(\frac{1}{2|b|^{2}}+\frac{r}{2}\right)}
$$

and then the problem (8) becomes

$$
\begin{aligned}
& \inf _{b}\left(\int\left(\frac{|b|^{2}}{8}+\frac{r}{2}\left(\nabla_{x} \phi^{n}-b\right) \cdot\left(\nabla_{x} \phi^{n}-b\right)+m^{n} \cdot\left(\nabla_{x} \phi^{n}-b\right)\right) d t d x\right. \\
& \left.+\int\left(c^{n} \partial_{t} \phi^{n}+\frac{r}{2}\left(\partial_{t} \phi^{n}\right)^{2}-\frac{\left(\frac{1}{2}-r \partial_{t} \phi^{n}-c^{n}\right)^{2}}{4\left(\frac{1}{2|b|^{2}}+\frac{r}{2}\right)}\right) d t d x\right) .
\end{aligned}
$$

Since we work in two space dimensions, we can use complex notations. Let us define $R=|b|$ and $\theta$ the phase of $b$ and let $\left(R_{0}, \theta^{0}\right)$ be defined by $m^{n}+r \nabla \phi^{n}=R_{0} e^{i \theta^{0}}$, problem (9) becomes

$$
\inf _{R \geq 0} \frac{R^{2}}{8}+\frac{r}{2}\left|R e^{i \theta}-R_{0} e^{i \theta^{0}}\right|^{2}-\frac{\left(\frac{1}{2}-r \partial_{t} \phi-c^{n}\right)^{2}}{2\left(\frac{1}{R^{2}}+r\right)}+C
$$

with $C$ staying constant. Since the term $\left|R e^{i \theta}-R_{0} e^{i \theta^{0}}\right|^{2}=\left|R e^{i \theta-\theta^{0}}-R_{0}\right|^{2} \geq\left|R-R_{0}\right|^{2}$, the optimal $b^{n}$ will be

$$
b^{n}=R^{*} \frac{m^{n}+r \nabla \phi^{n}}{\left|m^{n}+r \nabla \phi^{n}\right|}
$$

with $R^{*}$ the unique positive root of the polynomial

$$
Q(R)=\left(1+r R^{2}\right)^{2}\left(\frac{R}{4}+r\left(R-R_{0}\right)\right)-\left(\frac{1}{2}-r \partial_{t} \phi-c\right)^{2} R .
$$

The study of $Q(R)$ shows that $R^{*}$ is unique and that

$$
R^{*} \in\left[\frac{r R_{0}}{\frac{1}{4}+r} ; \max \left(\left(\frac{K}{r}\right)^{\frac{3}{2}} ; \frac{1+r R_{0}}{\frac{R}{4}+r\left(R-R_{0}\right)}\right)\right]
$$

with $K=\left|\frac{1}{2}-r \partial_{t} \phi-c\right|$. Indeed, the roots of the polynomial $Q(R)$ are also the solutions of the equation

$$
P(R)=\left(1+r R^{2}\right)^{2}\left(\frac{R}{4}+r\left(R-R_{0}\right)\right)=K^{2} R .
$$


The polynomial $P(R)$ has a unique real root

$$
\underline{R}=\frac{r R_{0}}{\frac{1}{4}+r} \leq R_{0} .
$$

Moreover, the equation (11) has at least a real positive solution because

$$
P(0)=-r R_{0} \leq 0 \text { and } P(R) \sim_{x \rightarrow \infty}\left(\frac{1}{4}+r\right) r^{2} R^{5} .
$$

This root is greater than $\underline{R}$ because $\forall R \leq \underline{R}, \quad P(R) \leq 0$. Since

$$
P^{\prime \prime}(R)=2\left(1+r R^{2}\right) 2 r R\left(\frac{R}{4}+r\left(R-R_{0}\right)\right)+\left(\frac{1}{4}+r\right)\left(1+r R^{2}\right)^{2}
$$

we have $P^{\prime \prime}(R) \geq 0$ for all $R \geq \underline{R}$. The equation (11) has then a unique positive solution because $P$ is strictly increasing (indeed $P^{\prime}$ is increasing and $P^{\prime}(\underline{R}) \geq 0$ ). Finally, we can show that the solution $R^{*}$ is bounded by $\bar{R}=\max \left(\left(\frac{K}{r}\right)^{\frac{3}{2}} ; \frac{1+r R_{0}}{\frac{R}{4}+r\left(R-R_{0}\right)}\right)$ because for $R \geq \bar{R}$ we have

$$
P(R) \geq r^{2} R^{4} \geq K^{2} R .
$$

We can then use any classical numerical method to look for this root. For programming simplicity, the numerical test showed here is made using a dichotomy method.

\subsection{Convergence criterium}

We deduce from (5) the formal optimality equations

$$
\partial_{t} \phi+\frac{1-2 c}{2}|\nabla \phi|^{2}=0
$$

with

$$
\frac{m}{c(1-c)}=\nabla \phi \text {. }
$$

We will use the numerical computation of $\left.\left|\partial_{t} \phi+\frac{1-2 c}{2}\right| \nabla \phi\right|^{2} \mid$ to decide if the program has converged. The history of the convergence will show the result of this numerical computation for each time. If this number is low enought, we will say that the program has converged. 


\section{Numerical TeST}

We present here a test made on the square discretised by $32 \times 32$ points on a time interval $[0, T]$ discretised by 32 points. The boundary conditions are periodic. We obtain the density at each point of the discretised square for each time.

The initial and final densities are the following (This case is in fact a translation)

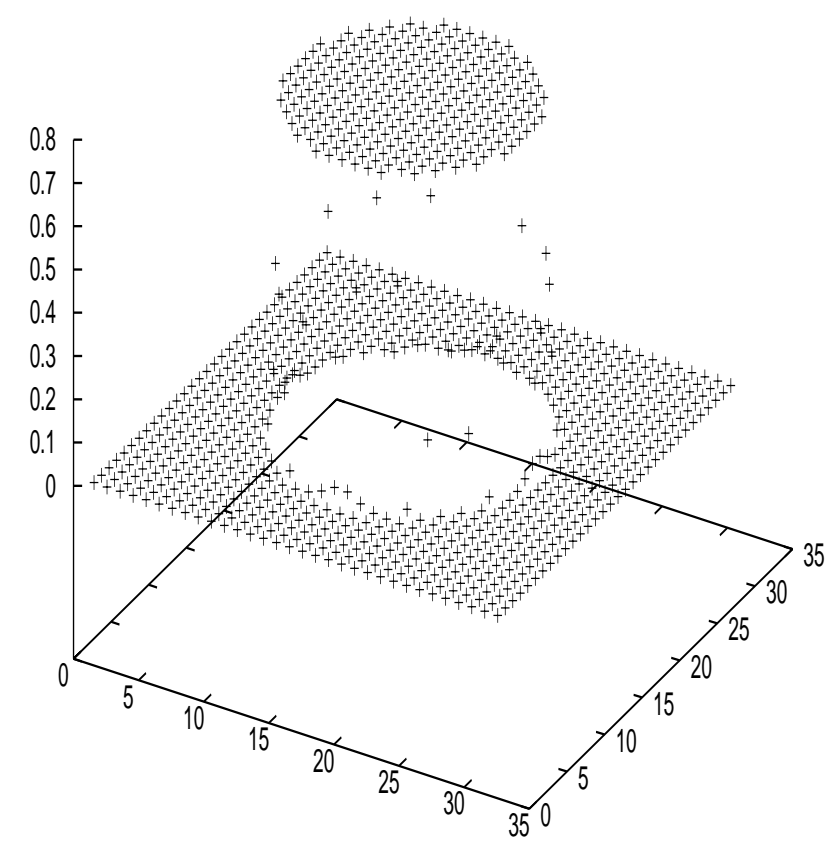

Initial density

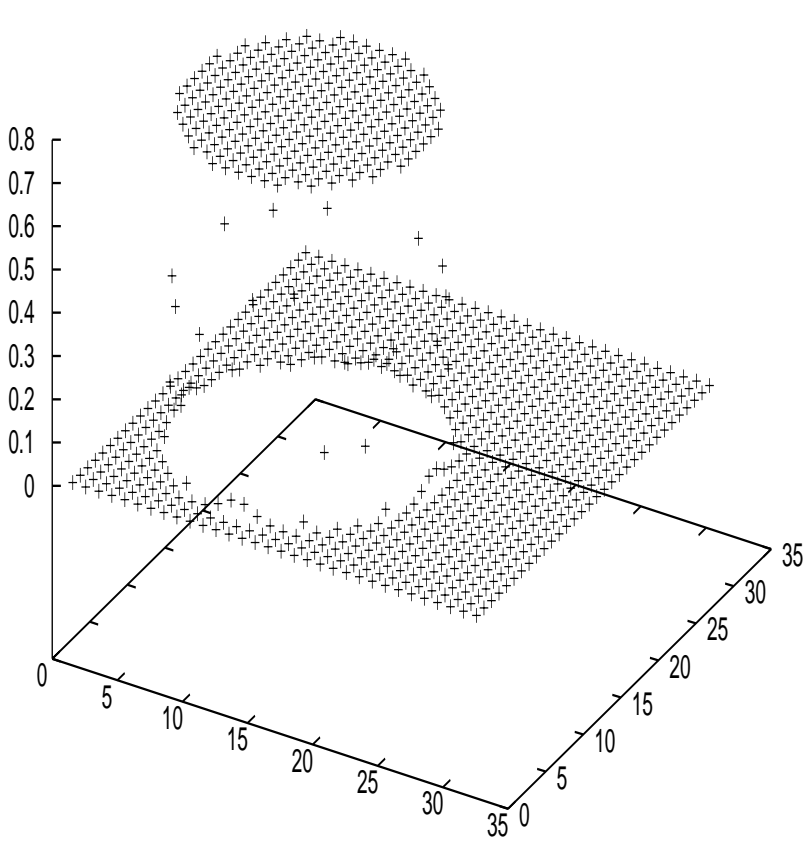

Final density

and we show the results after 50 iterations with a convergence history given by

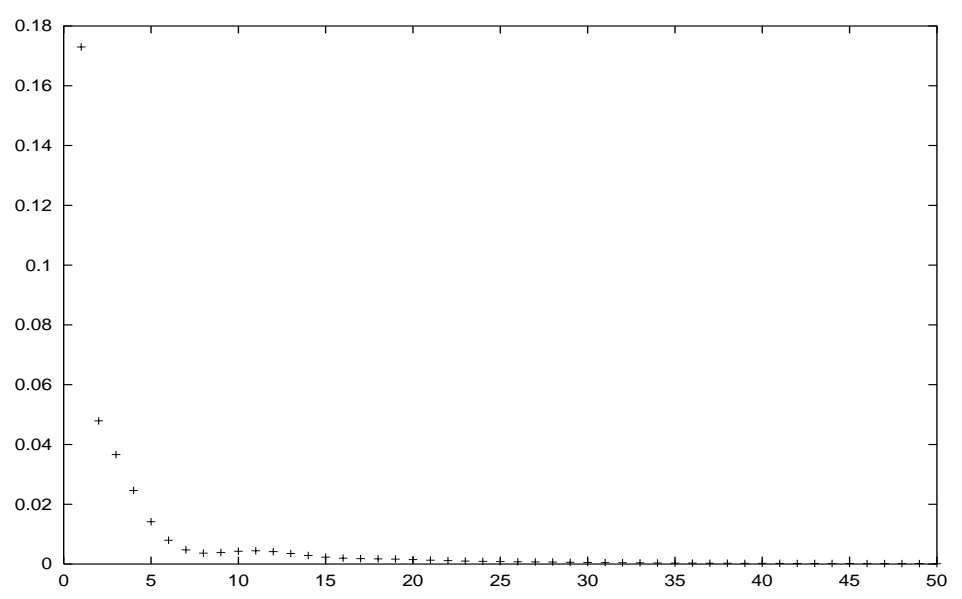

History of the convergence 
We then obtain the following density:

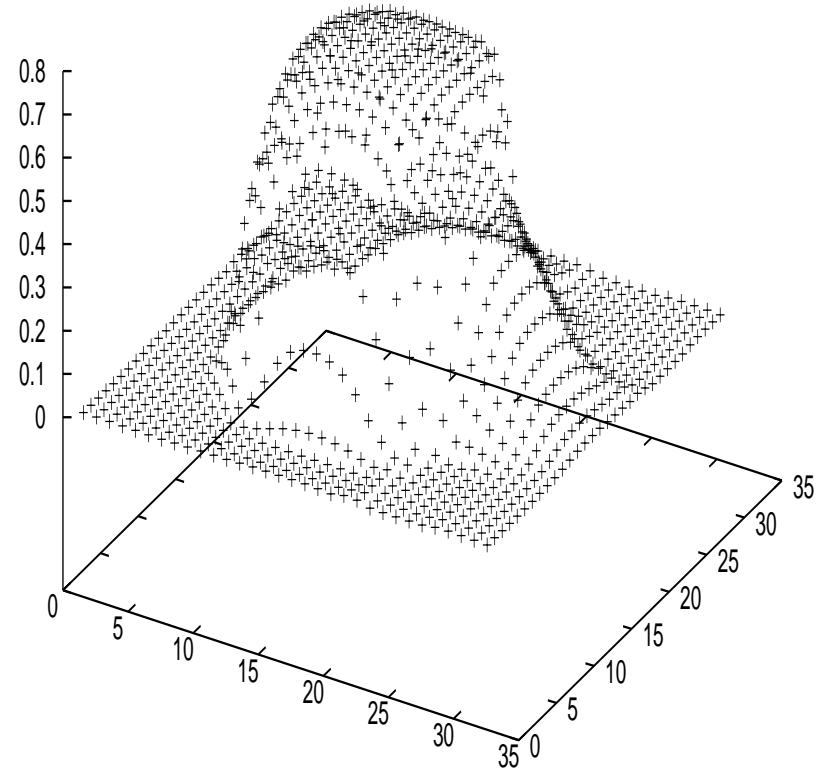

Density at time 6

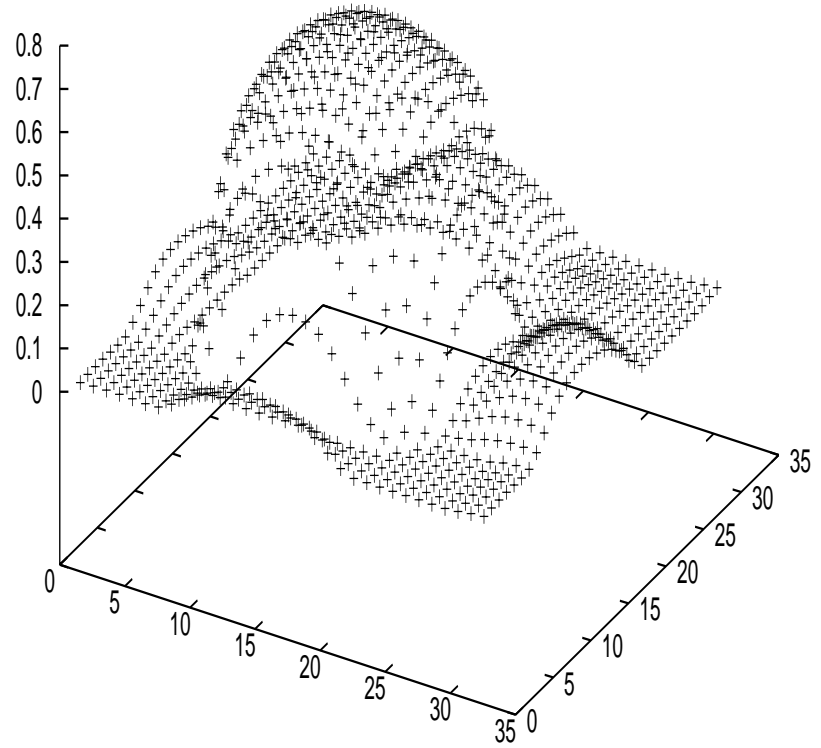

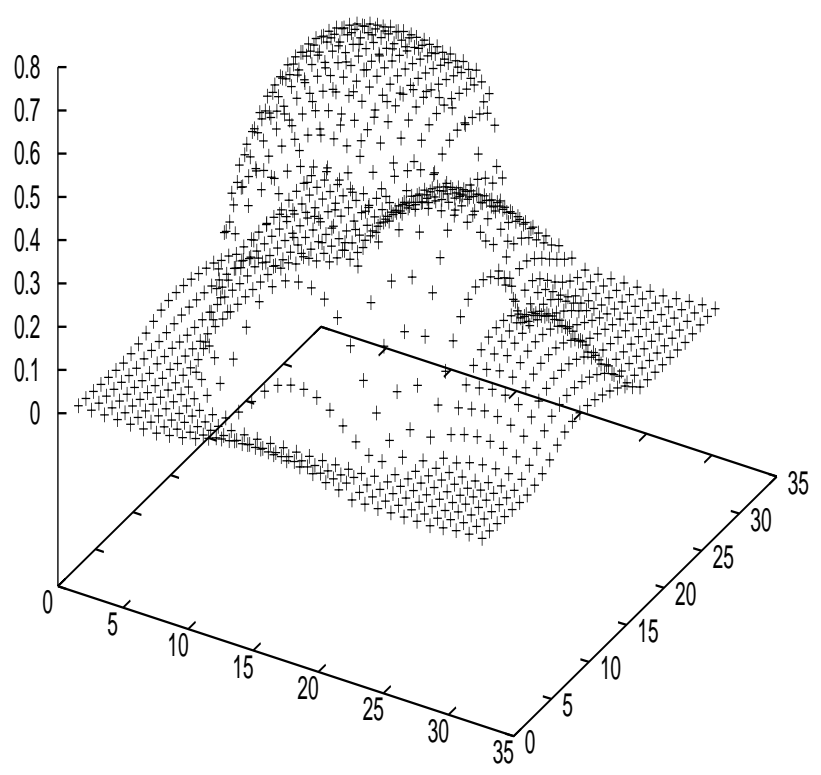

Density at time 10

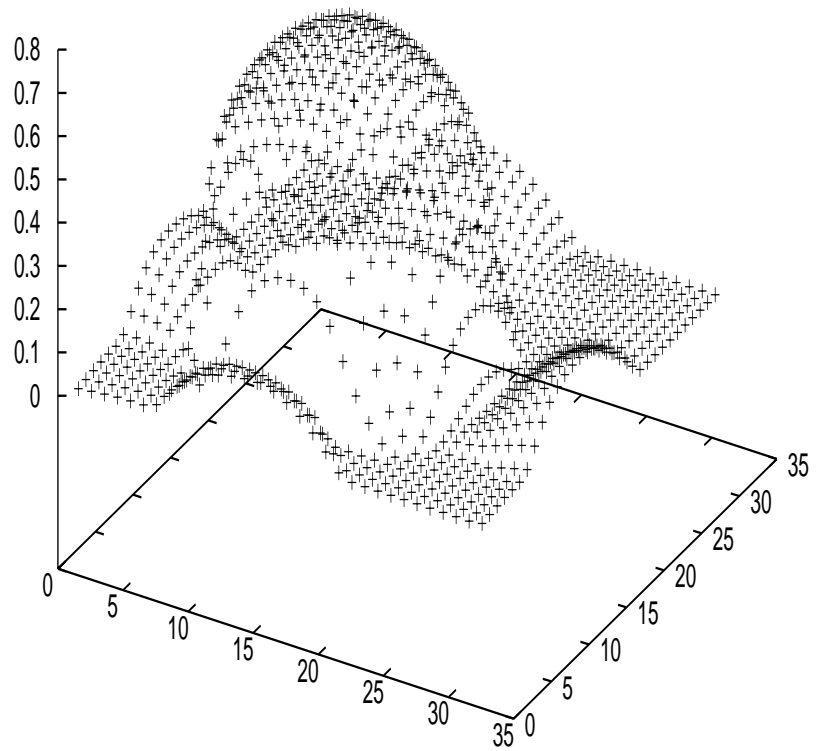




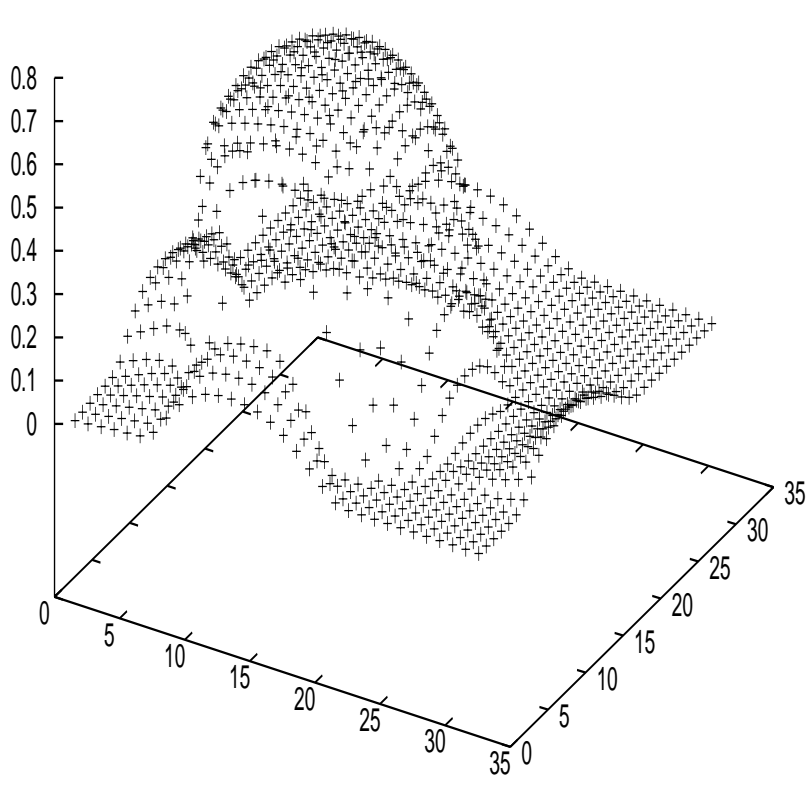

Density at time 22

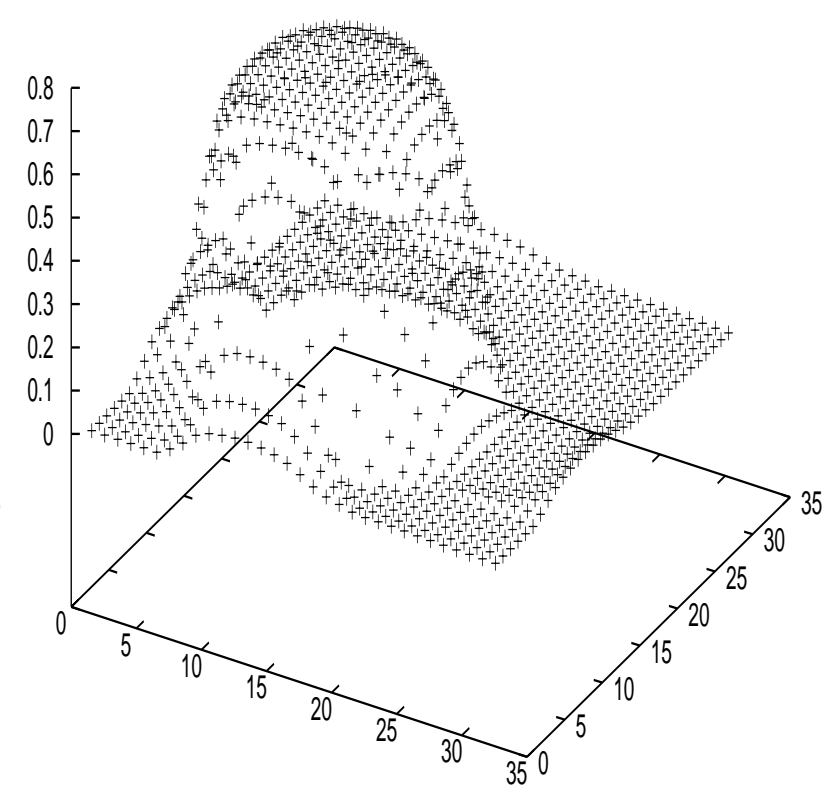

Density at time 26

\section{REFERENCES}

[BB] J.D. Benamou and Y.Brenier, A computational fluid mechanics solution to the Monge-Kantorovich mass transfer problem, Numer. Math. 84 (2000) 375-393.

[BR] Y. Brenier, Minimal geodesics on groups of volume-preserving maps, Comm. Pure Appl. Math. 52 (1999) $411-452$.

[FG] M. Fortin and R. Glowinski, Méthode du Lagrangien augmenté, Dunod.

[P] M. Puel, Construction variationnelle de flots multiphasiques avec vitesse moyenne imposée in preparation.

[S] R. Schlitzer, Determining the Mean, large-scale Circulation of the Atlantic with the adjoint Method, Journal of physical Oceanography, 23, 1993. 